\title{
Deep Learning Skin Disease Classifiers: Current Status and Future Prospects
}

\author{
Somesh Gupta, MD, DNB \\ Department of Dermatology \& Venereology, All India Institute of Medical Sciences, New Delhi, India
}

Corresponding Author:

Somesh Gupta, MD, DNB

Department of Dermatology \& Venereology

All India Institute of Medical Sciences

Sri Aurobindo Marg, Ansari Nagar

Ansari Nagar East

New Delhi

India

Phone: 911126588500

Email: someshgupta@ aiims.edu

\section{Abstract}

Background: Most studies on deep learning skin disease classifiers are done with binary classifications (ie, classifying lesions into malignant and benign). However, dermatology practice involves a large number of inflammatory and infective conditions that are not easily diagnosed by nondermatologist physicians.

Objective: The aim of this study is to develop a machine learning-based smartphone app for multiclass skin disease classification and evaluate its performance in different levels of dermatology practice. We will also explore similar studies in the literature.

Methods: We developed an artificial intelligence-driven smartphone app for 40 common skin diseases and tested it in primary care, tertiary care, and private practice settings.

Results: In the clinical study, the overall top-1 accuracy was 75.07\% (95\% CI 73.75\%-76.36\%), top-3 accuracy was $89.62 \%$ (95\% CI 88.67\%-90.52\%), and the mean area under the curve was 0.90 (SD 0.07). Multimedia Appendix 1 shows the top-1 positive predictive values and negative predictive values from a clinical study of 35 diseases using the developed mobile health app on patients. In the literature, there are very few studies on image-based deep learning multiclass classification of common skin diseases and none of them included evaluations in actual clinical settings.

Conclusions: An artificial intelligence-driven smartphone app has the potential to improve the diagnosis and management of skin diseases in patients with skin of color. Nondermatologist, primary care physicians are likely to benefit from having access to such an app.

Acknowledgments: Nurithm Labs collaborated with the All India Institute Of Medical Science to develop this smartphone app. Other collaborators in this work include Rashi Pangti, Jyoti Mathur, Vikas Chouhan, Sharad Kumar, Lavina Rajput, Sandesh Shah, Atula Gupta, Ambika Dixit, Dhwani Dholakia, Sanjeev Gupta, Savera Gupta, Mariam George, and Vinod Kumar Sharma.

Conflicts of Interest: None declared.

(iproc 2022;8(1):e36893) doi: $10.2196 / 36893$

\section{KEYWORDS}

artificial intelligence; deep learning; skin disease classifier; skin of color; mHealth; machine learning

\section{Multimedia Appendix 1}

Top-1 positive and negative predictive values from a clinical study of 35 diseases using a mobile health app on patients. [DOCX File, 17 KB-Multimedia Appendix 1] 
Edited by T Derrick; this is a non-peer-reviewed article. Submitted 28.01.22; accepted 28.01.22; published 15.02.22.

Please cite as:

Gupta $S$

Deep Learning Skin Disease Classifiers: Current Status and Future Prospects

iproc 2022;8(1):e36893

URL: https://www.iproc.org/2022/1/e36893

doi: $\underline{10.2196 / 36893}$

PMID:

CSomesh Gupta. Originally published in Iproceedings (https://www.iproc.org), 15.02.2022. This is an open-access article distributed under the terms of the Creative Commons Attribution License (https://creativecommons.org/licenses/by/4.0/), which permits unrestricted use, distribution, and reproduction in any medium, provided the original work, first published in Iproceedings, is properly cited. The complete bibliographic information, a link to the original publication on https://www.iproc.org/, as well as this copyright and license information must be included. 